JOURNAL OF

FUNCTION SPACES AND APPLICATIONS

Volume 7, Number 1 (2009), 1-12 (c) 2009, Scientific Horizon

http://www.jfsa.net

\title{
Boundedness of several operators on weighted Herz spaces
}

\author{
Yasuo Komori and Katsuo Matsuoka \\ (Communicated by Hans Triebel)
}

2000 Mathematics Subject Classification. 42B20.

Keywords and phrases. Herz space, singular integral, fractional integral, weight.

Abstract. We consider the boundedness of singular integral operators and fractional integral operators on weighted Herz spaces. For this purpose we introduce generalized Herz space. Our results are the best possible.

\section{Introduction}

Since Beurling [1] introduced the Beurling algebras and Herz [5] generalized these spaces, many studies have been done for Herz spaces (see, for example, [2], [4], [7] and [11]). Weighted Herz spaces are also considered in [6], [8] [9] and [10]. But they consider only $A_{1}$ weights or power weights.

In this paper we consider the boundedness of singular integral operators and fractional integral operators on weighted Herz spaces with $A_{p}$ weights. For this purpose, we introduce generalized Herz spaces, and as corollaries of our theorems we obtain the boundedness of these operators on weighted Herz spaces. 


\section{Definitions and Notation}

The following notation is used: For a set $E \subset R^{n}$ we denote the Lebesgue measure of $E$ by $|E|$. We denote the characteristic function of $E$ by $\chi_{E}$. We indicate a ball of radius $2^{k}$ centered at the origin by $B_{k}=\left\{x \in R^{n} ;|x| \leq 2^{k}\right\}$ where $k \in Z$. We write $C_{k}=B_{k} \backslash B_{k-1}$ and $\chi_{k}=\chi_{C_{k}}$. For a nonnegative function $w$, we write $w(E)=\int_{E} w(x) d x$ and define

$$
L^{p}(w)\left(R^{n}\right)=\left\{f ;\|f\|_{L^{p}(w)}=\left(\int_{R^{n}}|f(x)|^{p} w(x) d x\right)^{1 / p}<\infty\right\} .
$$

First we define homogeneous Herz spaces.

Definition 1 (Herz spaces). Let $1<p<\infty, 0<q<\infty$ and $-\infty<\alpha<\infty$. The ordinary Herz space is defined by

$$
K_{p}^{\alpha, q}\left(R^{n}\right)=\left\{f ;\|f\|_{K_{p}^{\alpha, q}}=\left(\sum_{k=-\infty}^{\infty} 2^{k \alpha q}\left\|f \chi_{k}\right\|_{L^{p}}^{q}\right)^{1 / q}<\infty\right\}
$$

For non-negative functions $w_{1}$ and $w_{2}$, weighted Herz space is defined by

$$
\begin{aligned}
K_{p}^{\alpha, q}\left[w_{1}, w_{2}\right]\left(R^{n}\right)=\left\{f ;\|f\|_{K_{p}^{\alpha, q}\left[w_{1}, w_{2}\right]}\right. & \\
& \left.=\left(\sum_{k=-\infty}^{\infty} w_{1}\left(B_{k}\right)^{\alpha q / n}\left\|f \chi_{k}\right\|_{L^{p}\left(w_{2}\right)}^{q}\right)^{1 / q}<\infty\right\}
\end{aligned}
$$

Next we define some classes of weight functions.

Definition 2. Let $1<p<\infty$. We say that $w \in A_{p}$ if for all balls $Q$

$$
\left(\frac{1}{|Q|} \int_{Q} w(x) d x\right)\left(\frac{1}{|Q|} \int_{Q} w(x)^{-1 /(p-1)} d x\right)^{p-1} \leq C .
$$

Definition 3. We say that $w \in A_{1}$ if for all balls $Q$

$$
\frac{1}{|Q|} \int_{Q} w(x) d x \leq C \text { ess } \inf _{x \in Q} w(x) .
$$

Remark . $A_{1} \subset A_{p}$ for all $p>1$.

Definition 4. Let $1<p_{1}, p_{2}<\infty$. We say that $w \in A\left(p_{1}, p_{2}\right)$ if

$$
\left(\frac{1}{|Q|} \int_{Q} w(x)^{p_{2}} d x\right)^{1 / p_{2}}\left(\frac{1}{|Q|} \int_{Q} w(x)^{-p_{1}^{\prime}} d x\right)^{1 / p_{1}^{\prime}} \leq C
$$


for all balls $Q$, where $1 / p_{1}+1 / p_{1}^{\prime}=1$.

Remarks . The following properties about weight functions are easily obtained from definitions. Especially the equivalence relation (1) is important for our theory (see Theorems 1 and 2).

$$
\begin{aligned}
& w \in A\left(p_{1}, p_{2}\right) \quad \text { if and only if } w^{p_{2}} \in A_{1+p_{2} / p_{1}^{\prime}} \\
& w \in A(p, p) \text { if and only if } w^{p} \in A_{p} . \\
& \text { If } \tilde{p}_{1}<p_{1} \text { and } \tilde{p}_{2}>p_{2} \text {, then } A\left(\tilde{p}_{1}, \tilde{p}_{2}\right) \subset A\left(p_{1}, p_{2}\right) .
\end{aligned}
$$

Definition 5 (centered reverse doubling). Let $\delta>0$. We say $w \in R D(\delta)$ if

$$
\frac{w\left(B_{k}\right)}{w\left(B_{j}\right)} \geq C 2^{\delta(k-j)} \quad \text { for } \quad k>j .
$$

The following lemmas are well-known (see, for example, [3] and [12]).

Lemma 1 (strong doubling). If $w \in A_{p}$, then

$$
\frac{w\left(B_{k}\right)}{w\left(B_{j}\right)} \leq C 2^{n p(k-j)} \quad \text { for } \quad k>j .
$$

Lemma 2. If $w \in A_{p}$, then $w \in R D(\delta)$ for some $\delta>0$.

Lemma 3. Let $1<p<\infty$. If $w \in A_{p}$, then $w \in A_{\tilde{p}}$ for some $\tilde{p}<p$.

Lemma 4. Let $1<p<\infty$ and $w=|x|^{\beta}$. Then $w \in A_{p}$ if and only if $-n<\beta<n(p-1)$.

Lemma 5. If $w \in A\left(p_{1}, p_{2}\right)$, then $w^{-p_{1}^{\prime}}(Q)^{1 / p_{1}^{\prime}} w^{p_{2}}(Q)^{1 / p_{2}} \leq$ $C|Q|^{1 / p_{1}^{\prime}+1 / p_{2}}$.

Remark . Note that $w^{p}(Q)^{1 / p}=\left(\int_{Q} w^{p}(x) d x\right)^{1 / p}$.

Next we define singular integral operators and fractional integral operators.

Definition 6. We say that $T$ is a standard singular integral operator if there exists a function $K$ which satisfies the following conditions.

$$
\begin{aligned}
& T f(x)=\mathrm{p} . \mathrm{v} \cdot \int_{R^{n}} K(x-y) f(y) d y . \\
& |K(x)| \leq \frac{C_{K}}{|x|^{n}} \quad \text { and } \quad|\nabla K(x)| \leq \frac{C_{K}}{|x|^{n+1}}, \quad x \neq 0 . \\
& \int_{\varepsilon<|x|<N} K(x) d x=0 \quad \text { for all } \quad 0<\varepsilon<N .
\end{aligned}
$$


Remark . We can weaken the conditions about $K$, but for the simplicity we assume these conditions.

Definition 7. The fractional integral operator is define by

$$
I_{s} f(x)=\int_{R^{n}} \frac{f(y)}{|x-y|^{n-s}} d y, \quad \text { where } \quad 0<s<n .
$$

\section{Known results}

Let $T$ be a standard singular integral operator and $I_{s}$ be the fractional integral operator. The following two propositions are well-known (see, for example, [12]).

Proposition 1. Let $1<p<\infty$. If $w \in A_{p}$, then $T$ is bounded on $L^{p}(w)$.

Proposition 2. Let $1<p_{1}<n / s$ and $1 / p_{2}=1 / p_{1}-s / n$. If $w \in A\left(p_{1}, p_{2}\right)$, then $I_{s}$ is bounded from $L^{p_{1}}\left(w^{p_{1}}\right)$ to $L^{p_{2}}\left(w^{p_{2}}\right)$.

Li and Yang [7] and Lu and Yang [9], [10] proved the next theorems about the boundedness on Herz space.

Theorem A ([7]). Let $1<p<\infty, 0<q<\infty$ and $-n / p<\alpha<$ $n(1-1 / p)$. Then $T$ is bounded on $K_{p}^{\alpha, q}$.

Theorem B ([9]). Let $1<p<\infty, 0<q<\infty$ and $0 \leq \alpha<n(1-1 / p)$. We assume that $w_{1}, w_{2} \in A_{1}$. Then $T$ is bounded on $K_{p}^{\alpha, q}\left[w_{1}, w_{2}\right]$.

Theorem C ([7]). Let $1<p_{1}<n / s, 0<q<\infty, 1 / p_{2}=1 / p_{1}-s / n$ and $-n / p_{1}+s<\alpha<n\left(1-1 / p_{1}\right)$. Then $I_{s}$ is bounded from $K_{p_{1}}^{\alpha, q}$ to $K_{p_{2}}^{\alpha, q}$.

Theorem D ([10]). Let $p_{1} \geq n / s, 0<q<n / s$ and

$$
-n / p_{1}+s<\alpha_{1}<n\left(1-1 / p_{1}\right)+s\left(1-q / p_{1}\right) .
$$

We set $1 / p_{2}=\left(1 / p_{1}\right) \cdot(1-q s / n)$ and $\alpha_{2}=\alpha_{1}+s\left(q / p_{1}-1\right)$. Then $I_{s}$ is bounded from $K_{p_{1}}^{\alpha_{1}, q}$ to $K_{p_{2}}^{\alpha_{2}, q}$.

$\mathrm{Lu}$ and Yang also proved weighted estimates for Theorems C and D.

Remark . In [10] p. 365, Lu and Yang assume the condition that $0<\alpha_{1}<n\left(1-1 / p_{1}\right)$ in stead of $(2)$, but this is incorrect. We shall show our condition (2) is the best possible in Section 7. Note that the condition (2) is equivalent to the following:

$$
-n / p_{2}<\alpha_{2}<n\left(1-1 / p_{1}\right) .
$$


In this paper we consider weighted estimates for these theorems with $A_{p}$ weights. For this purpose we introduce generalized Herz spaces in the next section, and as corollaries of our general theory, we can prove all the theorems above. We also prove that our results are the best possible by showing counterexamples in Section 7.

\section{Theorems}

For a sequence $\varphi=\{\varphi(k)\}_{k=-\infty}^{\infty}, \varphi(k)>0$, we define generalized Herz space as follows.

\section{Definition 8.}

$K_{p}^{q}(\varphi, w)\left(R^{n}\right)=\left\{f ;\|f\|_{K_{p}^{q}(\varphi, w)}=\left(\sum_{k=-\infty}^{\infty} \varphi(k)^{q}\left\|f \chi_{k}\right\|_{L^{p}(w)}^{q}\right)^{1 / q}<\infty\right\}$.

We define some conditions about $\varphi$.

Definition 9. Let $-\infty<a \leq b<\infty$. We say that $\varphi$ satisfies doubling condition of order $(a, b)$ and write $\varphi \in D(a, b)$, if there exists $C \geq 1$ such that

$$
C^{-1} 2^{a(k-j)} \leq \frac{\varphi(k)}{\varphi(j)} \leq C 2^{b(k-j)} \quad \text { for } \quad k>j .
$$

Definition 10. We say that $\varphi$ satisfies doubling condition, if $\varphi(k+1)+$ $\varphi(k-1) \leq C \varphi(k)$.

Remark . If $\varphi \in D(a, b)$, then $\varphi$ satisfies doubling condition.

Our results are the following.

Theorem 1. Let $1<p_{1}<n / s, 0<q<\infty, \delta>0$ and $1 / p_{2}=1 / p_{1}-s / n$. We assume that

$$
\begin{aligned}
& \varphi \in D(a, b), \quad \text { where } \quad-\frac{\delta}{p_{2}}<a \leq b<n\left(1-\frac{1}{p_{1}}\right), \\
& w^{p_{2}} \in A_{r}, \quad \text { where } \quad r=\min \left(1+\frac{p_{2}}{p_{1}^{\prime}}, 1+\frac{p_{2}}{p_{1}^{\prime}}-\frac{b p_{2}}{n}\right), \\
& w^{p_{2}} \in R D(\delta) .
\end{aligned}
$$

Then $I_{s}$ is bounded from $K_{p_{1}}^{q}\left(\varphi, w^{p_{1}}\right)$ to $K_{p_{2}}^{q}\left(\varphi, w^{p_{2}}\right)$.

Remark . When $b \leq 0$, the condition (4) is equivalent to the condition that $w \in A\left(p_{1}, p_{2}\right)$, but when $b>0$, (4) is stronger than $A\left(p_{1}, p_{2}\right)$ condition. We shall show that the condition (4) is the best possible by showing a counterexample in Section 7 . 
If we do not assume the condition (5), then in (3), we need to assume

$$
0 \leq a \leq b<n\left(1-\frac{1}{p_{1}}\right) .
$$

Theorem 2. Let $p_{1} \geq n / s, 0<q<n / s$ and $1 / p_{2}=\left(1 / p_{1}\right) \cdot(1-s q / n)$. We assume (3), (5) and the following:

(6) $w^{p_{2}} \in A_{r}, \quad$ where $r=\min \left(1+\frac{p_{2}}{p_{1}^{\prime}}, 1+\frac{p_{2}}{p_{1}^{\prime}}-\frac{p_{2}}{n}\left(b+s\left(1-\frac{q}{p_{1}}\right)\right)\right)$.

Then $I_{s}$ is bounded from $K_{p_{1}}^{q}\left(\psi, w^{p_{1}}\right)$ to $K_{p_{2}}^{q}\left(\varphi, w^{p_{2}}\right)$, where $\psi(k)=$ $2^{k s\left(1-q / p_{1}\right)} \varphi(k)$.

We also obtain the boundedness of singular integral operators.

Theorem 3. Let $T$ be a standard singular integral operator. Suppose that $1<p<\infty, 0<q<\infty$ and $\delta>0$. We assume that

$$
\begin{aligned}
& \varphi \in D(a, b), \quad \text { where } \quad-\frac{\delta}{p}<a \leq b<n\left(1-\frac{1}{p}\right), \\
& w \in A_{r}, \quad \text { where } \quad r=\min (p, p(1-b / n)), \\
& w \in R D(\delta) .
\end{aligned}
$$

Then $T$ is bounded from $K_{p}^{q}(\varphi, w)$ to $K_{p}^{q}(\varphi, w)$.

In Theorem 1, when we set $s=0$ formally, we can obtain Theorem 3 . The proof of Theorem 3 is similar to that of Theorem 1, therefore we omit the proof.

\section{Applications}

5.1 Applications to Theorems A, C and D. Let $\varphi(k)=2^{k \alpha_{2}}$ and $w \equiv 1$. Then $K_{p}^{q}(\varphi, w)=K_{p}^{\alpha_{2}, q}, \varphi \in D\left(\alpha_{2}, \alpha_{2}\right)$ and $w \in R D(n)$. Therefore the condition (3) in Theorem 1 is equivalent to the following:

$$
-\frac{n}{p_{2}}<\alpha_{2}<n\left(1-\frac{1}{p_{1}}\right) \text {. }
$$

Using Theorem 2, we obtain Theorem D. Let $\alpha_{1}=\alpha_{2}=\alpha$. Then we can prove Theorem C by Theorem 1. By Theorem 3, we obtain Theorem A.

5.2 Application to Theorem B. Suppose that $0 \leq \alpha<n(1-1 / p)$. For two weight functions $w_{1}, w_{2} \in A_{1}$, let $\varphi(k)=w_{1}\left(B_{k}\right)^{\alpha / n}$ and $w=w_{2}$. 
Then $K_{p}^{q}(\varphi, w)=K_{p}^{\alpha, q}\left[w_{1}, w_{2}\right], \varphi \in D(0, \alpha)$ and $w \in R D(\delta)$ for some $\delta>0$.

Since $\alpha(=b)$ satisfies the condition (3') in Theorem 3, we can prove Theorem B. Furthermore we obtain the following.

Corollary 1. Let $1<p<\infty, 0<q<\infty$ and $0 \leq \alpha<n(1-1 / p)$. If $w \in A_{p(1-\alpha / n)}$, then $T$ is bounded on $K_{p}^{\alpha, q}[1, w]$.

Remark . We shall prove that the condition that $w \in A_{p(1-\alpha / n)}$ is the best possible in Section 7 (see Counterexample 3).

Corollary 2. Let $1<p<\infty, 0<q<\infty$. We assume that

$$
-n<\alpha \leq 0 \quad \text { and } \quad-n-\alpha p<\beta<n(p-1)
$$

or

$$
0<\alpha<n(1-1 / p) \quad \text { and } \quad-n<\beta<n(p-1)-\alpha p .
$$

Then $T$ is bounded on $K_{p}^{\alpha, q}\left[1,|x|^{\beta}\right]$.

Proof of Corollary 2. Let $\varphi(k)=2^{k \alpha}$ and $w=|x|^{\beta}$. Then $\varphi \in D(\alpha, \alpha)$ and $w \in R D(n+\beta)$. Note that $w \in A_{r}$ if and only if $-n<\beta<n(r-1)$. Therefore the conditions (3') and (4') in Theorem 3 are equivalent to the following:

$$
\begin{aligned}
& -\frac{n+\beta}{p}<\alpha<n\left(1-\frac{1}{p}\right), \\
& -n<\beta<n(r-1) \quad \text { where } \quad r=\min (p, p-\alpha p / n) .
\end{aligned}
$$

These conditions are equivalent to (7) and (8).

5.3 Estimates for fractional integral operators (one weight case). The next two corollaries are easily obtained from Theorems 1 and 2 respectively.

Corollary 3. Let $1<p_{1}<n / s, 0<q<\infty, 0 \leq \alpha<n\left(1-1 / p_{1}\right)$ and $1 / p_{2}=1 / p_{1}-s / n$. If $w^{p_{2}} \in A_{1+p_{2} / p_{1}^{\prime}-\alpha p_{2} / n}$, then $I_{s}$ is bounded from $K_{p_{1}}^{\alpha, q}\left[1, w^{p_{1}}\right]$ to $K_{p_{2}}^{\alpha, q}\left[1, w^{p_{2}}\right]$.

Corollary 4. Let $p_{1} \geq n / s, 0<q<n / s, 0 \leq \alpha_{2}<n\left(1-1 / p_{1}\right)$, and we set $1 / p_{2}=1 / p_{1} \cdot(1-s q / n), \alpha_{2}=\alpha_{1}+s\left(q / p_{1}-1\right)$. If $w^{p_{2}} \in$

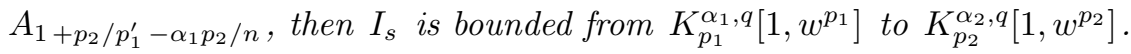

\section{Proofs}

To prove Theorems 1 and 2, we use the same calculation, therefore we first estimate $I_{s}$ for general indices $p_{1}, p_{2}$ and $q$. 
As in [7], we write

$$
\begin{aligned}
\left\|I_{s} f\right\|_{K_{p}^{q}(\varphi, w)} \leq & C\left(\sum_{k=-\infty}^{\infty} \varphi(k)^{q}\left(\sum_{j=-\infty}^{k-2}\left\|\left(I_{s}\left(f \chi_{j}\right)\right) \chi_{k}\right\|_{L^{p_{2}}\left(w^{p_{2}}\right)}\right)^{q}\right)^{1 / q} \\
& +C\left(\sum_{k=-\infty}^{\infty} \varphi(k)^{q}\left(\sum_{j=k-1}^{k+1}\left\|\left(I_{s}\left(f \chi_{j}\right)\right) \chi_{k}\right\|_{L^{p_{2}}\left(w^{p_{2}}\right)}\right)^{q}\right)^{1 / q} \\
& +C\left(\sum_{k=-\infty}^{\infty} \varphi(k)^{q}\left(\sum_{j=k+2}^{\infty}\left\|\left(I_{s}\left(f \chi_{j}\right)\right) \chi_{k}\right\|_{L^{p_{2}}\left(w^{p_{2}}\right)}\right)^{q}\right)^{1 / q} \\
= & I+I I+I I I .
\end{aligned}
$$

(I) When $j \leq k-2$ and $x \in C_{k}$, we have

$$
\left|I_{s}\left(f \chi_{j}\right)(x)\right| \leq C 2^{k(-n+s)} \int_{B_{j}}|f(y)| d y \leq C 2^{k(-n+s)}\left\|f \chi_{j}\right\|_{L^{p_{1}}\left(w^{p_{1}}\right)} w^{-p_{1}^{\prime}}\left(B_{j}\right)^{1 / p_{1}^{\prime}}
$$

and we obtain

$\left\|\left(I_{s}\left(f \chi_{j}\right)\right) \chi_{k}\right\|_{L^{p_{2}}\left(w^{p_{2}}\right)} \leq C 2^{k(-n+s)}\left\|f \chi_{j}\right\|_{L^{p_{1}}\left(w^{p_{1}}\right)} w^{-p_{1}^{\prime}}\left(B_{j}\right)^{1 / p_{1}^{\prime}} w^{p_{2}}\left(B_{k}\right)^{1 / p_{2}}$.

(III) When $j \geq k+2$ and $x \in C_{k}$, we have

$\left|I_{s}\left(f \chi_{j}\right)(x)\right| \leq C 2^{j(-n+s)} \int_{B_{j}}|f(y)| d y \leq C 2^{j(-n+s)}\left\|f \chi_{j}\right\|_{L^{p_{1}\left(w^{p_{1}}\right)}} w^{-p_{1}^{\prime}}\left(B_{j}\right)^{1 / p_{1}^{\prime}}$,

and we obtain

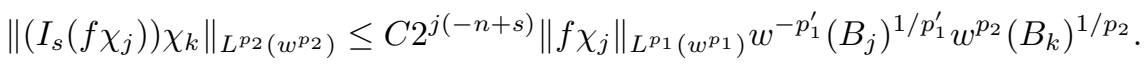

(I) By Lemma $3, w^{p_{2}} \in A_{\tilde{r}}$ for some $\tilde{r}<r$. Therefore by Lemmas 1 and 5 , we have

$$
\begin{aligned}
& \left\|\left(I_{s}\left(f \chi_{j}\right)\right) \chi_{k}\right\|_{L^{p_{2}}\left(w^{p_{2}}\right)} \\
& \quad \leq C 2^{k(-n+s)}\left\|f \chi_{j}\right\|_{L^{p_{1}}\left(w^{p_{1}}\right)} w^{-p_{1}^{\prime}}\left(B_{j}\right)^{1 / p_{1}^{\prime}} w^{p_{2}}\left(B_{j}\right)^{1 / p_{2}}\left(\frac{w^{p_{2}}\left(B_{k}\right)}{w^{p_{2}}\left(B_{j}\right)}\right)^{1 / p_{2}} \\
& \quad \leq C 2^{k\left(-n+s+n \tilde{r} / p_{2}\right)} 2^{n j\left(1 / p_{1}^{\prime}+1 / p_{2}-\tilde{r} / p_{2}\right)}\left\|f \chi_{j}\right\|_{L^{p_{1}}\left(w^{p_{1}}\right)} .
\end{aligned}
$$

(III) By Lemma $2, w^{p_{2}} \in R D(\delta)$ for some $\delta>0$. Therefore by the same argument as above we have

$$
\left\|\left(I_{s}\left(f \chi_{j}\right)\right) \chi_{k}\right\|_{L^{p_{2}}\left(w^{p_{2}}\right)} \leq C 2^{k \delta / p_{2}} 2^{j\left(-n+s+n / p_{1}^{\prime}+n / p_{2}-\delta / p_{2}\right)}\left\|f \chi_{j}\right\|_{L^{p_{1}}\left(w^{p_{1}}\right)} .
$$


Using the condition that $\varphi \in D(a, b)$, we obtain the following inequalities.

$$
\begin{aligned}
I^{q} \leq & C \sum_{k} 2^{k\left(-n+s+n \tilde{r} / p_{2}+b\right) q} \\
& \times\left(\sum_{j \leq k-2} 2^{n j\left(1 / p_{1}^{\prime}+1 / p_{2}-\tilde{r} / p_{2}-b / n\right)} \varphi(j)\left\|f \chi_{j}\right\|_{L^{p_{1}}\left(w^{p_{1}}\right)}\right)^{q}, \\
I I I^{q} \leq & C \sum_{k} 2^{k\left(a+\delta / p_{2}\right) q} \\
& \times\left(\sum_{j \geq k+2} 2^{j\left(-n+s+n / p_{1}^{\prime}+n / p_{2}-\delta / p_{2}-a\right)} \varphi(j)\left\|f \chi_{j}\right\|_{L^{p_{1}}\left(w^{p_{1}}\right)}\right)^{q} .
\end{aligned}
$$

These are elementary estimates for I and III. From here we shall prove two theorems respectively.

Proof of Theorem 1. The estimate of II is easy. By using Proposition 2, we obtain II $\leq C\|f\|_{K_{p_{1}\left(\varphi, w^{p_{1}}\right)} \text {. }}$.

Next we estimate I. When $q \leq 1$, we have

$$
\begin{aligned}
I^{q} \leq & C \sum_{j} 2^{n j\left(1 / p_{1}^{\prime}+1 / p_{2}-\tilde{r} / p_{2}-b / n\right) q} \varphi(j)^{q} \\
& \quad \times\left\|f \chi_{j}\right\|_{L^{p_{1}}\left(w^{\left.p_{1}\right)}\right.}^{q}\left(\sum_{k=j+2}^{\infty} 2^{k\left(-n+s+n \tilde{r} / p_{2}+b\right) q}\right) \\
\leq C & \|f\|_{K_{p}^{q}\left(\varphi, w^{p_{1}}\right)},
\end{aligned}
$$

because $-n+s+n r / p_{2}+b \leq 0$, that is, $-n+s+n \tilde{r} / p_{2}+b<0$.

When $q>1$, we take $\varepsilon>0$ such that $-n+s+n \tilde{r} / p_{2}+b+n \varepsilon<0$. Then we have

$$
\begin{aligned}
I^{q} \leq C & \sum_{k} 2^{k\left(-n+s+n \tilde{r} / p_{2}+b\right) q} \\
& \times\left(\left(\sum_{j=-\infty}^{k-2} 2^{n j\left(1 / p_{1}^{\prime}+1 / p_{2}-\tilde{r} / p_{2}-b / n-\varepsilon\right) q} \varphi(j)^{q}\left\|f \chi_{j}\right\|_{L^{p_{1}}\left(w^{p_{1}}\right)}^{q}\right)\right. \\
& \left.\times\left(\sum_{j=-\infty}^{k-2} 2^{n \varepsilon q^{\prime} j}\right)^{q / q^{\prime}}\right) \\
\leq & C \sum_{k} 2^{k\left(-n+s+n \tilde{r} / p_{2}+b+n \varepsilon\right) q} \\
& \times\left(\sum_{j=-\infty}^{k-2} 2^{n j\left(1 / p_{1}^{\prime}+1 / p_{2}-\tilde{r} / p_{2}-b / n-\varepsilon\right) q} \varphi(j)^{q}\left\|f \chi_{j}\right\|_{L^{p_{1}}\left(w^{p_{1}}\right)}^{q}\right)
\end{aligned}
$$


$\leq C\|f\|_{K_{p}^{q}\left(\varphi, w^{p_{1}}\right)}$.

Finally we estimate $I I I$. When $q \leq 1$, we have

$$
\begin{aligned}
I I I^{q} \leq & C \sum_{j} 2^{j\left(-n+s+n / p_{1}^{\prime}+n / p_{2}-\delta / p_{2}-a\right) q} \varphi(j)^{q}\left\|f \chi_{j}\right\|_{L^{p_{1}}\left(w^{p_{1}}\right)}^{q} \\
& \times\left(\sum_{k=-\infty}^{j-2} 2^{k\left(a+\delta / p_{2}\right) q}\right) \\
\leq & C\|f\|_{K_{p}^{q}\left(\varphi, w^{p_{1}}\right)}
\end{aligned}
$$

because $a+\delta / p_{2}>0$.

When $q>1$, we take $\varepsilon>0$ such that $a+\delta / p_{2}-\varepsilon>0$. Then we have

$$
\begin{aligned}
I I I^{q} \leq & C \sum_{k} 2^{k\left(a+\delta / p_{2}\right) q} \\
& \times\left(\left(\sum_{j \geq k+2}^{\infty} 2^{j\left(-n+s+n / p_{1}^{\prime}+n / p_{2}-\delta / p_{2}-a+\varepsilon\right)} \varphi(j)^{q}\left\|f \chi_{j}\right\|_{L^{p_{1}}\left(w^{p_{1}}\right)}\right)\right. \\
& \left.\times\left(\sum_{j=k+2}^{\infty} 2^{-j q^{\prime} \varepsilon}\right)^{q / q^{\prime}}\right) \\
\leq & C\|f\|_{K_{p}^{q}\left(\varphi, w^{p_{1}}\right)} .
\end{aligned}
$$

Proof of Theorem 2. We can estimate I and III same as above (note that $\left.-n+s+n r / p_{2}+b \leq 0\right)$, therefore we omit the proof. We shall estimate II. We can take $\tilde{p_{1}}$ and $\tilde{p_{2}}$ such that $1 / \tilde{p}_{2}=1 / \tilde{p}_{1}-s / n, \tilde{p}_{1}<n / s$ and $\tilde{p}_{2}>p_{2}$ (take $\tilde{p}_{1}$ sufficiently near $\left.n / s\right)$. Then $w \in A\left(\tilde{p}_{1}, \tilde{p}_{2}\right)$, therefore we can apply Proposition 2 to these indices. We have

$$
\begin{aligned}
\left\|\left(I_{s}\left(f \chi_{j}\right)\right) \chi_{k}\right\|_{L^{p_{2}}\left(w^{p_{2}}\right)} & \leq\left|B_{k}\right|^{1 / p_{2}-1 / \tilde{p}_{2}}\left\|\left(I_{s}\left(f \chi_{j}\right)\right)\right\|_{L^{\tilde{p}_{2}}\left(w^{\tilde{p}_{2}}\right)} \\
& \leq C\left|B_{k}\right|^{1 / p_{2}-1 / \tilde{p}_{2}}\left\|f \chi_{j}\right\|_{L^{\tilde{p}_{1}}\left(w^{\tilde{p}_{1}}\right)} \\
& \leq C\left|B_{k}\right|^{1 / p_{2}-1 / \tilde{p}_{2}}\left|B_{j}\right|^{1 / \tilde{p}_{1}-1 / p_{1}}\left\|f \chi_{j}\right\|_{L^{p_{1}}\left(w^{p_{1}}\right)} \\
& \leq C 2^{j s\left(1-q / p_{1}\right)}\left\|f \chi_{j}\right\|_{L^{p_{1}\left(w^{p_{1}}\right)} .}
\end{aligned}
$$

This proves the theorem.

\section{Counterexamples}

In this section we shall show that the condition (2') in Theorem D and the condition that $w \in A_{p(1-\alpha / n)}$ in Corollary 1 are the best possible. 
For the simplicity we consider the case when $n=1$. Let $H$ be the Hilbert transform $H f(x)=\int_{R^{1}} f(y) /(x-y) d y$ and $I_{s} f(x)=\int_{R^{1}} f(y) /|x-y|^{1-s} d y$.

Counterexample 1. Theorem $D$ is not true if $\alpha_{2} \leq-1 / p_{2}$.

First, note that $\chi_{[0,1]} \notin K_{p_{2}}^{\alpha_{2}, q}\left(R^{1}\right)$ when $\alpha_{2} \leq-1 / p_{2}$.

Let $f=\chi_{[1,2]}$, then $f \in K_{p_{1}}^{\alpha_{1}, q}\left(R^{1}\right)$.

But $I_{s} f(x) \geq C>0$ when $0<x<1$, therefore $I_{s} f \notin K_{p_{2}}^{\alpha_{2}, q}\left(R^{1}\right)$

Counterexample 2. Theorem $D$ is not true if $\alpha_{2} \geq 1-1 / p_{1}$.

If $\alpha_{2} \geq 1-1 / p_{1}$, then $\alpha_{1} \geq 1-1 / p_{1}+s\left(1-q / p_{1}\right)>0$, because $q<p_{1}$. Therefore $f=\chi_{[0,1]} \in K_{p_{1}}^{\alpha_{1}, q}\left(R^{1}\right)$.

On the contrary, $I_{s} f(x) \geq C 2^{k(-1+s)}$ where $2^{k-1}<x<2^{k}$ and $k \geq 2$. We have

$$
\left\|I_{s} f\right\|_{K_{p_{2}}^{\alpha_{2}, q}}^{q} \geq C \sum_{k=2}^{\infty} 2^{k q\left(\alpha_{2}-1+s+1 / p_{2}\right)}=\infty,
$$

because $\alpha_{2}-1+s+1 / p_{2} \geq s\left(1-q / p_{1}\right)>0$.

Counterexample 3. Corollary 1 is not true if $w \in A_{r}$ where $r \geq p(1-\alpha)$.

Let $r=p(1-\alpha)+\varepsilon$, where $\varepsilon$ is a sufficiently small positive number, and we define $\beta=r-1-\varepsilon / 2=p(1-\alpha)-1+\varepsilon / 2$.

By Lemma $4, w=|x|^{\beta} \in A_{r}$.

For $k<1$, let $f_{k}(x)=\chi_{\left[2^{k-1}, 2^{k}\right]}(x)$. Then we have

$$
\left\|f_{k}\right\|_{K_{p}^{\alpha, q}[1, w]} \leq C 2^{k(\alpha+(\beta+1) / p)}=C 2^{k(1+\varepsilon / 2 p)} .
$$

On the contrary, $H f_{k}(x) \geq C 2^{k}$ where $1<x<2$. Therefore we have

$$
\left\|H f_{k}\right\|_{K_{p}^{\alpha, q}[1, w]} \geq C 2^{k} .
$$

By (13) and (14), we know that $H$ is not bounded on $K_{p}^{\alpha, q}[1, w]\left(R^{1}\right)$.

\section{References}

[1] A. Beurling, Construction and analysis of some convolution algebras, Ann. Inst. Fourier Grenoble, 14 (1964), 1-32.

[2] J. García-Cuerva, Hardy spaces and Beurling algebras, J. London Math. Soc., 39 (1989), 499-513.

[3] L. Grafakos, Classical and Modern Fourier Analysis, Pearson Education, New Jersey, 2004. 
[4] E. Hernández and D. Yang, Interpolation of Herz-type Hardy spaces, Illinois J. Math., 42 (1998), 564-581.

[5] C. Herz, Lipschitz spaces and Bernstein's theorem on absolutely convergent Fourier transforms, J. Math. Mech., 18 (1968), 283-324.

[6] Y. Komori, Weak type estimates for Calderón-Zygmund operators on Herz spaces at critical indexes, Math. Nachr., 259 (2003), 42-50.

[7] X. Li and D. Yang, Boundedness of some sublinear operators on Herz spaces, Illinois J. Math., 40 (1996), 484-501.

[8] S. Z. Lu and F. Soria, On the Herz spaces with power weights, Fourier Analysis and Partial Differential Equations, CRC Press, (1995), 227236.

[9] S. Z. Lu and D. Yang, The decomposition of weighted Herz space on $R^{n}$ and its applications, Sci. in China, 38 (1995), 147-158.

[10] S. Z. Lu and D. Yang, Hardy-Littlewood-Sobolev theorems of fractional integration on Herz-type spaces and its applications, Canad. J. Math., 48 (1996), 363-380.

[11] S. Z. Lu and D. Yang, The continuity of commutators on Herz-type spaces, Michigan Math. J., 44 (1997), 255-281.

[12] A. Torchinsky, Real-Variable Methods in Harmonic Analysis, Academic Press, 1986.

School of High Technology for Human Welfare

Tokai University

317 Nishino Numazu

Shizuoka 410-0395

Japan

(E-mail : komori@wing.ncc.u-tokai.ac.jp)

College of Economics, Nihon University

1-3-2, Misaki-cho Chiyoda-ku

Tokyo 101-8360

Japan

(E-mail : katsu@eco.nihon-u.ac.jp)

(Received : May 2007) 


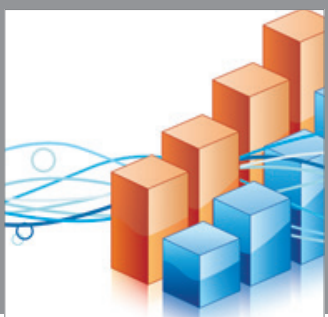

Advances in

Operations Research

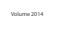

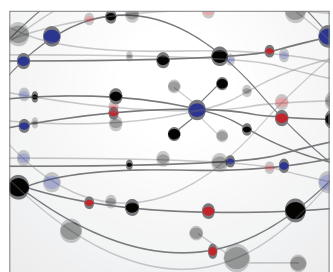

\section{The Scientific} World Journal
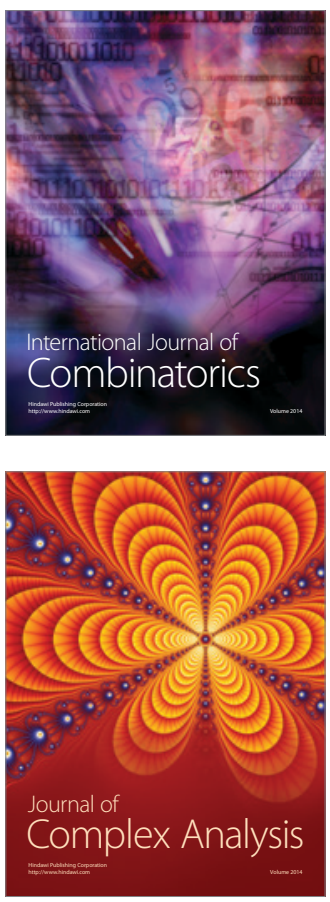

International Journal of

Mathematics and

Mathematical

Sciences
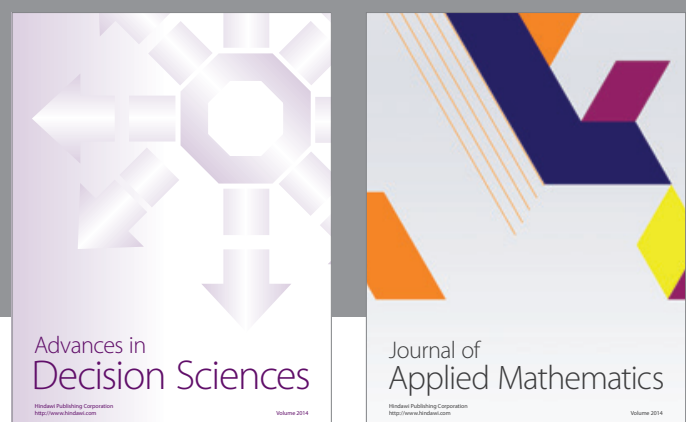

Journal of

Applied Mathematics
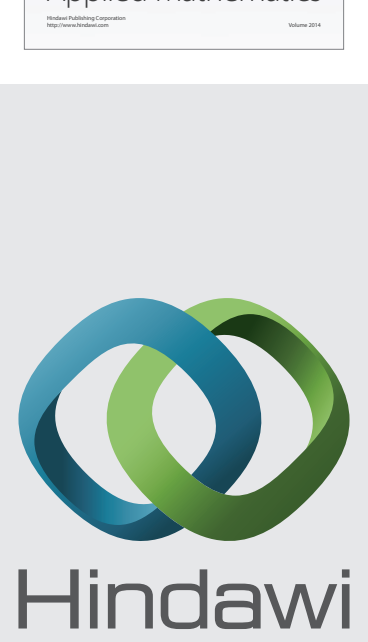

Submit your manuscripts at http://www.hindawi.com
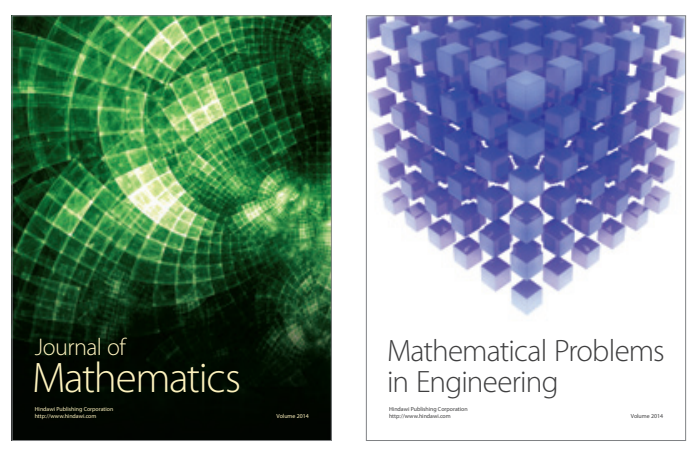

Mathematical Problems in Engineering
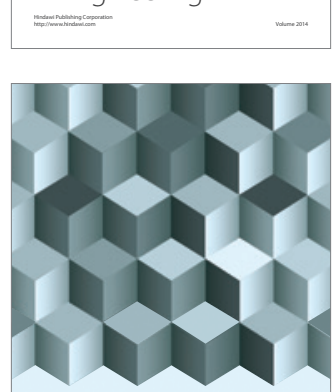

Journal of

Function Spaces
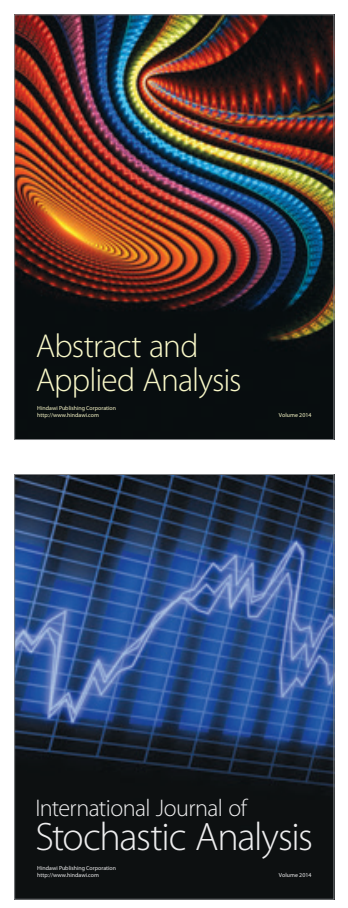

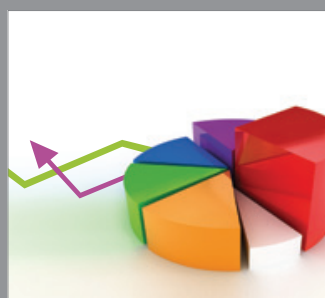

ournal of

Probability and Statistics

Promensencen
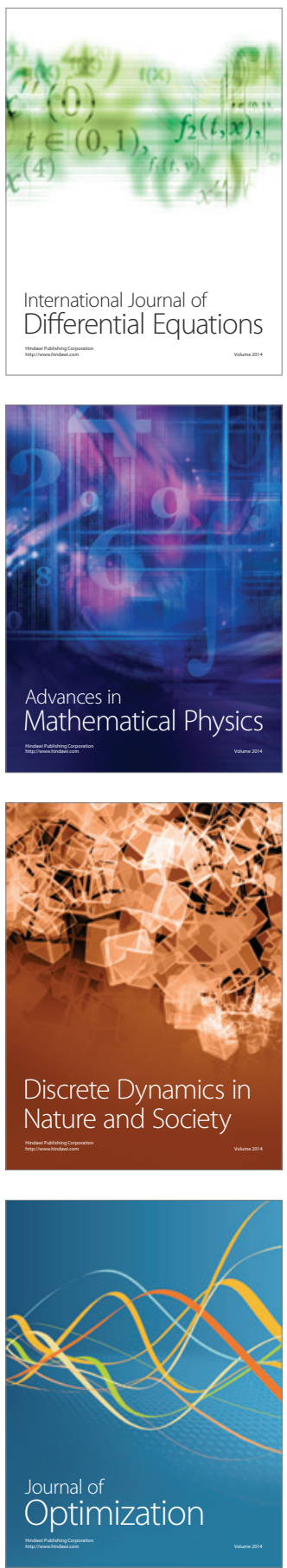